\title{
Table of cited WTO reports
}

Argentina - Hides and Leather: Argentina-Measures Affecting the Export of Bovine Hides and the Imports of Finished Leather, Panel Report, WT/ DS155/R and Corr.1, 19 December 2000, adopted 16 February 2001 123

Brazil-Retreaded Tyres: Brazil - Measures Affecting Imports of Retreaded Tyres, Panel Report, WT/DS332/R, 12 June 2007, modified by Appellate Body Report, WT/DS332/AB/R, 3 December 2007; Article 21.3 (c) Arbitration Report, 29 August 2008, WT/DS332/16, adopted 20 August 2009 ........ 151-4,

$168,184,283-4$

Canada-Autos: Canada-Certain Measures Affecting the Automotive Industry, Panel Report, WT/DS139/R, WT/DS142/R, 11 February 2000, modified by Appellate Body Report, 31 May 2000, WT/DS139/AB/R, WT/DS142/AB/R, adopted 19 June 2000 96, 120, 138-9

Canada-Feed-in Tariff Program: Canada-Measures Relating to the Feed-in Tariff Program, Panel Report, WT/DS412/R, WT/DS426/R, 19 December 2012, modified by Appellate Body Report, 6 May 2013, WT/DS412/AB/R, WT/ DS426/AB/R, adopted 24 May 2013

Canada - Periodicals: Canada - Certain Measures Concerning Periodicals, Panel Report,WT/DS31/R and Corr.1, 14 March 1997, modified by Appellate Body Report, 30 June 1997, WT/DS31/AB/R, adopted 30 July 1997 116,133

Chile-Alcoholic Beverages: Chile - Taxes on Alcoholic Beverages, Panel Report, WT/DS87/R, WT/DS110/R, 15 June 1999, modified by Appellate Body Report, 13 December 1999, WT/DS87/AB/R, WT/DS110/AB/R, adopted 12 January 2000 126,134

China-Auto Parts: China - Measures Affecting Imports of Automobile Parts, Panel Report, WT/DS339/R, WT/DS340/R, WT/DS342/R, 18 July 2008, upheld by Appellate Body Report, 15 December 2008, WT/DS339/AB/R, and modified by Appellate Body Report, 15 December 2008, WT/DS340/AB/R, WT/ DS342/AB/R, adopted 12 January 2009 $80,82-3,217$

China-Publications and Audiovisual Products: China-Measures Affecting Trading Rights and Distribution Services for Certain Publications and Audiovisual Entertainment Products, Panel Report, WT/DS363/R, 12 August 2009, upheld by Appellate Body Report, 21 December 2009, WT/DS363/AB/R, adopted 19 January 2010

China-Raw Materials: China-Measures Related to the Exportation of Various Raw Materials, Panel Report, WT/DS394/R, WT/DS395/R, WT/DS398/R, 5 July 2011, modified by Appellate Body Report, 30 January 2012, WT/DS394/ AB/R, WT/DS395/AB/R, WT/DS398/AB/R, adopted 22 February 2012 . 147, $155-6,217$ 
Colombia - Ports of Entry: Colombia - Indicative Prices and Restrictions on Ports of Entry, Panel Report, WT/DS366/R, 27 April 2009, adopted 20 May 2009

Dominican Republic - Import and Sale of Cigarettes: Dominican Republic Measures Affecting the Importation and Internal Sale of Cigarettes, Panel Report, WT/DS302/R, 26 November 2004, modified by Appellate Body Report, 25 April 2005, WT/DS302/AB/R, adopted 19 May 2005 $123-4,129,135,183$

EC-Approval and Marketing of Biotech Products: European Communities Measures Affecting the Approval and Marketing of Biotech Products, Panel Report, WT/DS291/R, WT/DS292/R and WT/DS293/R, 29 September 2006, adopted 21 November 2006 $40,112,136,246$

EC - Asbestos: European Communities - Measures Affecting Asbestos and Asbestos Containing Products, Panel Report, WT/DS135/R and Add.1, 18 September 2000, modified by Appellate Body Report, 12 March 2001, WT/ DS135/AB/R, adopted 5 April 2001 ...... 29, 76, 87, 109-12, 118-19, 121, 126, $135,142,150-4,176-7,183,192-3,197$

EC - Bananas III: European Communities - Regime for the Importation, Sale and Distribution of Bananas, Panel Report, Complaint by Ecuador, WT/DS27/R/ ECU, by Mexico, WT/DS27/R/MEX, by the United States, WT/DS27/R/ USA, and by Guatemala and Honduras, WT/DS27/R/GTM and WT/DS27/R/ HND, 22 May 1997, modified by Appellate Body Report, 9 September 1997, WT/DS27/AB/R, adopted 25 September 1997 ....... 123, 126, 134-5, 150, 193,

EC-Bananas III - Article 21.5 (Ecuador): European Communities - Regime for the Importation, Sale and Distribution of Bananas, Second Recourse to Article 21.5 of the DSU by Ecuador, Panel Report, WT/DS27/RW2/ECU, 7 April 2008, modified by Appellate Body Report, 26 November 2008, WT/DS27/ AB/RW2/ECU, adopted 11 December 2008

EC-Hormones: EC Measures Concerning Meat and Meat Products (Hormones), Panel Report, 18 August 1997, Complaint by Canada, WT/DS48/R/CAN, and by the United States, WT/DS26/R/USA, modified by Appellate Body Report, 16 January 1998, WT/DS26/AB/R, WT/DS48/AB/R, adopted 13 February 1998

EC - Sardines: European Communities - Trade description of Sardines, Panel Report, WT/DS231/R, 29 May 2002, modified by Appellate Body Report, 26 September 2002, WT/DS231/AB/R, adopted 23 October 2002 .... 141, 190-91

EC - Tariff Preferences: European Communities - Conditions for the Granting of Tariff Preferences to Developing Countries, Panel Report, WT/DS246/R, 1 December 2003, modified by Appellate Body Report, 7 April 2004, WT/ DS246/AB/R, adopted 20 April 2004

India-Additional Import Duties: India - Additional and Extra-Additional Duties on Imports from the United States, WT/DS360/R, 9 June 2008, modified by Appellate Body Report, 30 October 2008, WT/DS360/AB/R, adopted 17 November 2008 $73-5,80,84$

Indonesia - Autos: Indonesia - Certain Measures Affecting the Automobile Industry, Panel Report, WT/DS54/R, WT/DS55/R, WT/DS59/R, WT/DS64/R and Corr.1, 2, 3, and 4, 2 July 1998, adopted 23 July 1998 ..... 120, 138-9 
Japan - Alcoholic Beverages II: Japan - Taxes on Alcoholic Beverages, Panel Report, WT/DS8/R, WT/DS10/R, WT/DS11/R, 11 July 1996, modified by Appellate Body Report, 4 October 1996, WT/DS8/AB/R, WT/DS10/AB/R, WT/DS11/AB/R, adopted 1 November 1996 109, 114-18, 122, 126-7, $131-3,251$

Korea-Alcoholic Beverages: Korea - Taxes on Alcoholic Beverages, Panel Report, WT/DS75/R, WT/DS84/R, 17 September 1998, modified by Appellate Body Report, 18 January 1999, WT/DS75/AB/R, WT/DS84/AB/R, adopted 17 February 1999 $116-17,133$

Korea - Various Measures on Beef: Korea - Measures Affecting Imports of Fresh, Chilled and Frozen Beef, Panel Report, WT/DS161/R, WT/DS169/R, 31 July 2000, modified by Appellate Body Report, 11 December 2000, WT/DS161/ AB/R, WT/DS169/AB/R, adopted 10 January 2001 ........ 76, 129, 135, 151-2,

Mexico - Taxes on Soft Drinks: Mexico - Tax Measures on Soft Drinks and Other Beverages, Panel Report, WT/DS308/R, 7 October 2005, modified by Appellate Body Report, 6 March 2006, WT/DS308/AB/R WT/DS308/R, adopted 24 March 2006

Philippines - Distilled Spirits: Philippines - Taxes on Distilled Spirits, Panel Report, WT/DS396/R, WT/DS403/R, 15 August 2011, modified by Appellate Body Report, 21 December 2011, WT/DS396/AB/R, WT/DS403/AB/R, adopted 20 January 2012 110-11, 116-17

Shrimp/Turtle see US-Shrimp

Shrimp/Turtle - Article 21.5 see US/Shrimp - Article 21.5

Thailand-Cigarettes (Philippines): Thailand-Customs and Fiscal Measures on Cigarettes from the Philippines, Panel Report, WT/DS371/R, 15 November 2010, modified by Appellate Body Report, 17 June 2011, WT/DS371/AB/R, adopted 15 July 2011

Turkey-Textiles: Turkey - Restrictions on Imports of Textile and Clothing Products, Panel Report, WT/DS34/R, 31 May 1999, modified by Appellate Body Report, 22 October 1999, WT/DS34/AB/R, adopted 19 November 1999 $280,284,286$

US - Clove Cigarettes: United States - Measures Affecting the Production and Sale of Clove Cigarettes, Panel Report, WT/DS406/R, 2 September 2011, upheld by Appellate Body Report, 4 April 2012, WT/DS406/AB/R, adopted 24 April 2012 120-1, 124, 127-8, 142-4, 147, 187, 192-3, 254

US - COOL: United States - Certain Country of Origin Labelling (COOL) Requirements, Panel Report, WT/DS384/R and WT/DS386/R, 18 November 2011, modified by Appellate Body Report, 29 June 2012, WT/DS384/AB/R and WT/DS386/AB/R, adopted 23 July 2012 .................................. 144, 192

US - FSC: United States - Tax Treatment for 'Foreign Sales Corporations', Panel Report, WT/DS108/R, 8 October 1999, modified by Appellate Body Report, 24 February 2000, WT/DS108/AB/R, adopted 20 March 2000 ....... 24, 202-3,

US - FSC (Article 21.5 - EC): United States - Tax Treatment for 'Foreign Sales Corporations- Recourse to Article 21.5 of the DSU by the European Communities, Panel Report, WT/DS108/RW, 20 August 2001, modified by 
Appellate Body Report, 14 January 2002, WT/DS108/AB/RW, adopted 29 January 2002 119,134

US - Gambling: United States - Measures Affecting the Cross-Border Supply of Gambling and Betting Services, Panel Report, WT/DS285/R, 10 November 2004, modified by Appellate Body Report, 7 April 2005, WT/DS285/AB/R, adopted 20 April 2005 $151-3,167$

US - Gasoline: United States - Standards for Reformulated and Conventional Gasoline, Panel Report, WT/DS2/R, 29 January 1996, modified by Appellate Body Report, 29 April 1996, WT/DS2/AB/R, adopted 20 May 1996 $32-4$, $114,150-1,155-6,166-8,176,191$

US - Shrimp: United States - Import Prohibition of Certain Shrimp and Shrimp Products, Panel Report, WT/DS58/R and Corr.1, 15 May 1998, modified by Appellate Body Report, 12 October 1998, WT/DS58/AB/R, adopted 6 November 1998 ....29, 34, 96-7, 148-9, 154-5, 164, 166-71, 173-5, 180, 195

US - Shrimp - Article 21.5: United States - Import Prohibition of Certain Shrimp and Shrimp Products - Recourse to Article 21.5 of the DSU by Malaysia, Panel Report, WT/DS58/RW, 15 June 2001, upheld by Appellate Body Report, 22 October 2001, WT/DS58/AB/RW, adopted 21 November 2001 ........... 154,

$161-2,166-7,169,177,180,195$

US - Softwood Lumber IV: United States - Countervailing Duty Determination with Respect to Certain Softwood Lumber from Canada, Panel Report, WT/ DS257/R and Corr.1, 29 August 2003, modified by Appellate Body Report, 19 January 2004, WT/DS257/AB/R, adopted 17 February 2004 193,212

US - Tuna II (Mexico): United States - Measures Concerning the Importation, Marketing and Sale of Tuna and Tuna Products, Panel Report, WT/DS381/R, 15 September 2011, modified by the Appellate Body Report, 16 May 2012, WT/DS381/AB/R, adopted in June 2012 .97, 112-13, 124, 129, 141-5, $164-5,188-90,192-3,197$ 